\title{
A low-cost multimode fiber Bragg grating sensor system
}

\author{
Tongyu Liu ${ }^{\mathrm{a}}$, Daorui Wang ${ }^{\mathrm{b}}$, Rasool Raenaei ${ }^{\mathrm{a}}$, Xianfeng Chen ${ }^{\mathrm{c}}$, Lin Zhang ${ }^{\mathrm{c}}$, Ian Bennion ${ }^{\mathrm{c}}$ \\ ${ }^{a}$ Intelligent Sensor Systems Ltd, Brunel Science Park, Uxbridge, Middx UB8 3PQ UK \\ ${ }^{\mathrm{b}}$ Shandong Laser Institute, SDAS, 123 Hong Xing Dong Lu, Jining, Shandong, 272017 PRC \\ ${ }^{c}$ Photonics Research Group, Aston University, Aston Triangle, Birmingham, B4 7ET UK
}

\begin{abstract}
A low-cost fiber optic sensor system based on multimode fiber and an LED light source is presented. A multimode fiber Bragg grating (MMFBG) element is used as a strain sensor. In a matched grating scheme, a MMFBG similar to the sensing one was used as a reference in the receiving unit. For detection of large wavelength shift we demonstrated the feasibility of MMFBG wavelength detection using a single mode fiber fused coupler edge filter. The high cost normally associated with wavelength interrogators for single mode fiber FBG sensors was overcome by the utilization of a low cost multimode fiber pigtailed LED light source. The multimode fiber sensing system has the potential of maintaining much of the advantages of its single mode FBG sensor system counterparts. The MMFBG sensing schemes could be used for short distance, high sensitivity, high speed, strain, temperature and acoustic sensing applications.
\end{abstract}

Keywords: Fiber Bragg grating, multimode, sensor, edge filter, passive detection.

\section{INTRODUCTION}

Optical fiber sensors (OFS) have been increasingly accepted over the past few years in applications where conventional electronic sensors suffer serious limitations. They are particularly advantageous in hazardous environment such as high electromagnetic fields, high radiation, corrosive, flammable or explosive environment. Among various sensor technologies, fiber Bragg grating (FBG) has been especially attractive due to its wavelength encoding nature, high mechanical strength and small size. FBGs have been used to construct a variety of sensors such as temperature ${ }^{1}$, strain ${ }^{2}$, acceleration $^{3}$, pressure ${ }^{4,5,6}$ and current sensors ${ }^{7}$. The fundamental principle of FBG signal processing is to detect its characteristic wavelength value or wavelength shift. This can be achieved typically by using a tunable laser ${ }^{8}$, a tunable optical filter $^{9}$, an interferometer ${ }^{2}$ and optical edge filters ${ }^{10,11,12}$ or matched FBG filters ${ }^{13}$. One of the major advantages of FBG sensor systems is its capability for large scale multiplexing. Wavelength division, time division and spatial division multiplexing and their combinations are commonly used in medium capacity sensor network. A very large capacity low reflectivity FBG sensor system based on high-resolution photon counting-OTDR has also been reported ${ }^{14}$. Whilst FBG sensors are advantageous in many aspects over their electronic counterparts, their relatively high cost of the optical signal interrogators present a major limitation in many applications, even though its per sensor cost could be competitive in a large capacity multiplexing system. This is particularly the case where the number of sensors required is not very large. For example, in a temperature monitoring system for an electrical transformer, it normally requires only a few sensors to monitor the temperature of the winding coil and the oil. It will be difficult to justify the cost associated with the implementation of a tunable laser based sensor system as the large multiplexing capacity provided by such system is excessive for such application. Furthermore in many industrial monitoring and control applications the distance between the sensors and the interrogator is not very long which does not require the high optical power level from tunable laser or the amplified spontaneous emission (ASE) source. Therefore it is of great importance to develop low cost, high 
performance OFS solutions for the small sensor count, short distance applications in order to make the FBG sensor systems more competitive to conventional technologies both in the industrial and the domestic applications.

An FBG interrogator typically has two basic functionalities: i) wavelength discrimination and ii) sensor conditioning including provision of optical power. With regard to the system cost, for the tunable laser based systems, the cost of wavelength discrimination i.e. the control and monitoring mechanisms for laser wavelength tuning is dominant. For the tunable filter based system, the hardware cost is mainly due to the optical power such as the ASE source and the tunable optical filter. Therefore the cost of the FBG interrogator may be reduced by using (i) passive detection schemes instead of any tuning device and (ii) using multimode fibers instead of single mode fibers to construct both the fiber link and the FBG sensor elements. As the multimode fiber has a larger core and larger N.A. than those of the single mode fibers, it can coupled light more efficiently from low cost broadband LED light sources.

The application for bend sensing using MMFBGs have been proposed theoretically analyzed by Wanser et al. ${ }^{15}$, where the modulation of the reflection spectrum profile was used to correlate with the bending angle of the fiber lead prior to the sensing grating element. Characterization of MMFBGs made of standard graded-index 50/125um fibers and a few mode FBGs made of dispersion shifted fibers as well as experimental investigation of the temperature and bending effect using the MMFBGs were reported by Mizunami et al ${ }^{16}$. The feasibility of strain and temperature sensing using MMFBGs was demonstrated by Zhao and Claus. ${ }^{17}$ To address the issue of multi-peak discrimination characteristic of the MMFBG sensors, Lim et al ${ }^{18}$ reported a signal processing method based on the correlation between the reflection spectrum from the sensor and a pre-recorded reference spectrum from the same sensor. To the knowledge of the authors, there has been no report to date on the wavelength discrimination of MMFBG sensors based on passive wavelength discrimination such as matched grating filters and edge filters, or using low cost LED light sources.

\section{EXPERIMENTAL}

\subsection{Principle of the multimode fiber Bragg gratings}

When a multimode fiber is subject to axial periodic index modulation with a spatial period of $\Lambda$, the phase matching or Bragg reflection condition for a specific spatial mode is $\beta_{1}-\beta_{2}=2 \pi / \Lambda$, where $\beta_{1}$ and $\beta_{2}$ are propagation constants of the forward and backward mode. The standard multimode fiber can guide hundreds of modes for the wavelengths around 1310 or $1550 \mathrm{~nm}$. Since some of them have almost identical propagation constants, they manifest themselves as a number of degenerated principal modes ${ }^{16}$, corresponding to a series of different Bragg reflection wavelengths. In multimode fiber, the propagation constant for the $\mathrm{N}^{\text {th }}$ principal mode, $\beta_{N}$, can be described by:

$$
\beta_{N}=\frac{2 \pi n_{1}}{\lambda} \sqrt{1-\frac{4 \Delta(N+1)}{V}}
$$

where $n_{l}$ is the core refractive index, $\Delta$ the maximum relative index difference, $V=2 \pi a N A / \lambda$ the normalized frequency, $a$ the core radius, and $N A$ the numerical aperture.

The phase matching conditions can be satisfied both between reflection of the same modes and the neighboring modes. For the reflection between the same $\mathrm{N}^{\text {th }}$ principal mode, the Bragg wavelength is determined by $\lambda=2 n_{1} \Lambda(\sqrt{(1-4 \Delta(N+1) / V})$. For the matching conditions between the $\mathrm{N}^{\text {th }}$ and the $(\mathrm{N}+1)^{\text {th }}$ reflection modes, the Bragg wavelength is determined by the average value of the two neighboring propagation constants, $\beta=\beta_{\mathrm{N}}+\beta_{\mathrm{N}+1}$, hence the peak reflection will fall between the $\mathrm{N}^{\text {th }}$ and $(\mathrm{N}+1)^{\text {th }}$ reflection modes.

The number of principal modes or Bragg wavelengths depends on the how many modes have been excited in the fiber. Light from broadband white light sources can excite all the fiber modes hence all the principal modes. More than 20 Bragg wavelengths can be observed for multimode excitation. When single mode fiber pigtailed narrower band light sources such as the SLDs are used to illuminate the multimode fiber, not all the modes can be excited. Consequently smaller number of Bragg wavelengths can be observed than in the multimode excitation case. 
Similar to single mode fiber FBGs, the reflection wavelengths of the MMFBGs can be modulated by both the ambient temperature and axial strain. The wavelength shift due to strain for a specific principal mode can be described by

$$
\Delta \lambda_{B}=\left(1-P_{e}\right) \lambda_{B} \varepsilon
$$

where $\lambda_{B}$ and $\Delta \lambda_{B}$ are Bragg wavelength and the wavelength shift respectively when applied with axial strain $\varepsilon$, $P_{e}$ is the effective photoelastic coefficient. Two approaches have been adopted in this work for wavelength detection. The $1^{\text {st }}$ one is based on a matched MMFBG, the $2^{\text {nd }}$ approach is based on an edge filter.

\subsection{Wavelength discrimination scheme based on matched MMFBGs}

Two batches of the MMFBGs were fabricated using standard 50/125um graded-index multimode fibers and used in this experimental work. The gratings were fabricated using the same procedure as established for the single mode fibers. The fibers were prepared under $\mathrm{H}_{2}$ loading at 100 atmospheres for two weeks. The interferometric method was used to fabricate the gratings, such that the central wavelengths of the fundamental modes were around 1310 or $1550 \mathrm{~nm}$ respectively, which were the nominal peak wavelengths of the fiber pigtailed LED light sources. The $1310 \mathrm{~nm}$ LED was pigtailed with 50/125 um multimode fibers and the $1550 \mathrm{~nm}$ LED was pigtailed with a 9/125um single mode fibers.

Two gratings were selected at each wavelength to carry out the experiment. The experimental arrangement is shown in Figure 1. A multimode fiber pigtailed LED (model no. LED341, supplied by WTD, China) was connected to a 50:50 multimode fiber coupler. The LED had a spectral bandwidth (FWHM) of $\sim 40 \mathrm{~nm}$, output power of $50 \mu \mathrm{W}$. The sensing grating was connected to one output arm of the coupler. The other output was terminated with index matching gel to eliminate Fresnel reflection from the fiber tip. To vary the grating wavelength, the sensing grating was stretched using a precision micrometer. The reflected light from the sensing grating was connected to the input of the second multimode coupler. A reference grating which had a similar characteristics as the sensing one was connected to one output arm of the second coupler. The other output arm was connected to an optical spectrum analyzer (OSA) (HP6142A). The reflection from the second reference grating was connected to a multimode fiber pigtailed InGaAs photodiode. The optical signal was converted to electrical signal and amplified via a trans-impedance amplifier (TIA) circuit. Two MMFBG pairs which had wavelengths at $\sim 1310$ and $\sim 1550 \mathrm{~nm}$ respectively were evaluated.

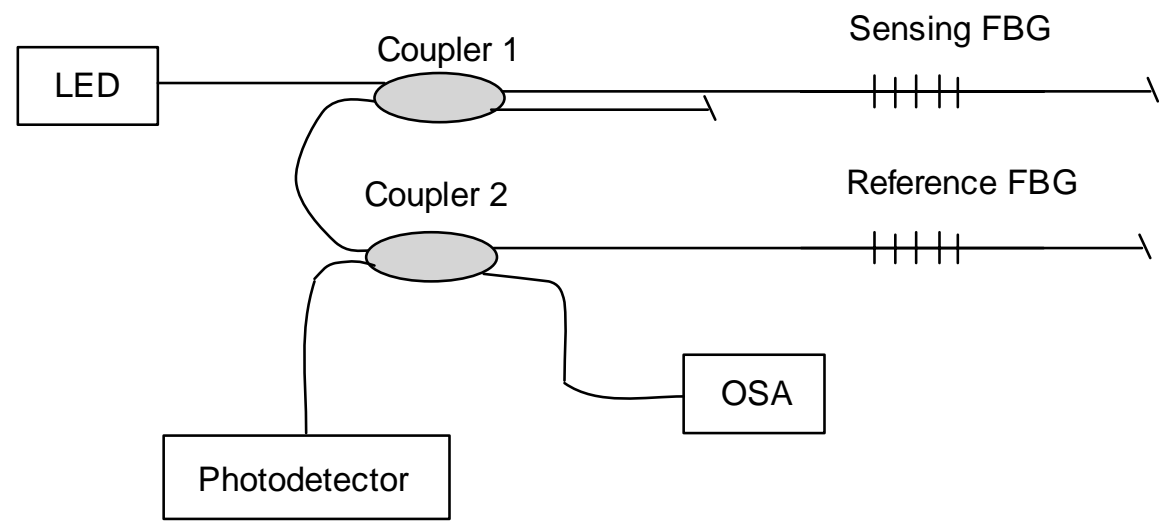

Figure 1 Experimental arrangement for matched filter detection.

\subsection{Wavelength discrimination based on an edge filter}

The experimental arrangement of the edge filter based wavelength discrimination scheme is illustrated in Figure 2. Light from a $1550 \mathrm{~nm}$ single mode fiber pigtailed LED was connected to a $3 \mathrm{~dB}$ multimode coupler. A MMFBG sensing grating was connected to one output arm of the coupler. The grating was stretched using a precision micrometer. The second output port of the coupler was monitored using an OSA. The reflected signal from the sensing grating was connected to an over-coupled, fused single mode fiber coupler. The fused coupler had a characteristic tilted spectral response at around $1550 \mathrm{~nm}$, hence acted as an edge filter. The photodiode was connected to the output arm which had a positive slope with the increase of the wavelength and amplified using a TIA circuit. The other output arm was not being used in this experiment, although it could be connected to another detector. The peak wavelengths of the recorded spectra using the OSA when the sensing grating was stretched were analyzed and correlated with the output signal level of the TIA circuit. 


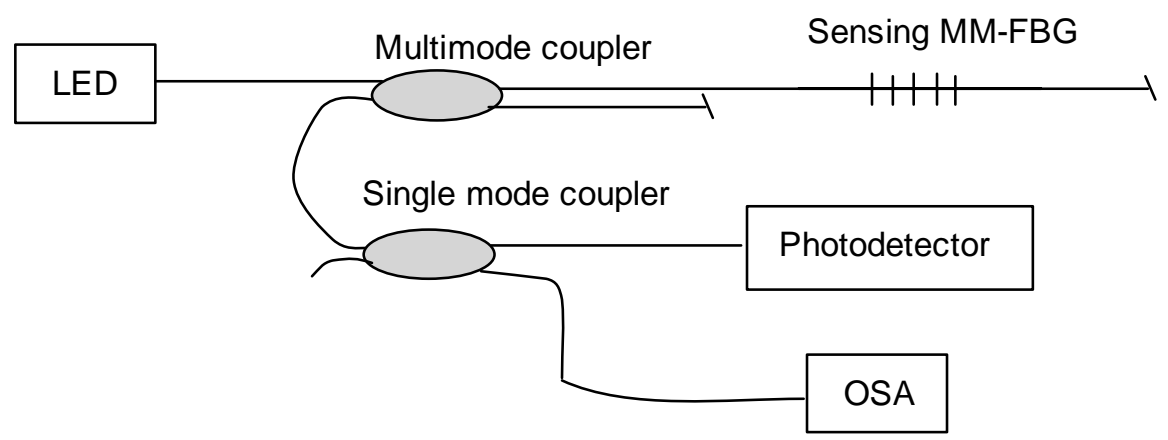

Figure 2 The experimental arrangement of the edge filter detection.

\section{RESULTS AND DISCUSSIONS}

\subsection{The matched MMFBG scheme.}

The spectra of the two matched MMFBGs with fundamental wavelengths at around $1310 \mathrm{~nm}$ are shown in Figure 3. Grating \#18 was used as a sensor and \#17 as the reference. More than 20 peaks can be observed in the spectra due to multimode propagation from the multimode fiber pigtailed LED source. When the sensing grating is applied with tension, its wavelengths will increase as a result the overlapping area between the sensing and the reference gratings will decrease. Hence the detected signal by the photodiode was expected to decrease.

The correlation between the peak reflection wavelengths (the $2^{\text {nd }}$ mode) of the sensing MMFBG spectra and the detected power reflected by the reference FBG is shown in Figure 4. Signal varied from $30 \mathrm{mV}$ to $180 \mathrm{mV}$ for a wavelength change of approximately $0.13 \mathrm{~nm}$. The sensitive region is around $1309.60 \mathrm{~nm}$, and the linear region is approximately 1909.53 to $1509.67 \mathrm{~nm}$ or $+-0.07 \mathrm{~nm}$. When the reference grating was stabilized at the sensitive operation region, sub- $\mu \varepsilon$ can be achieve using this discrimination scheme.

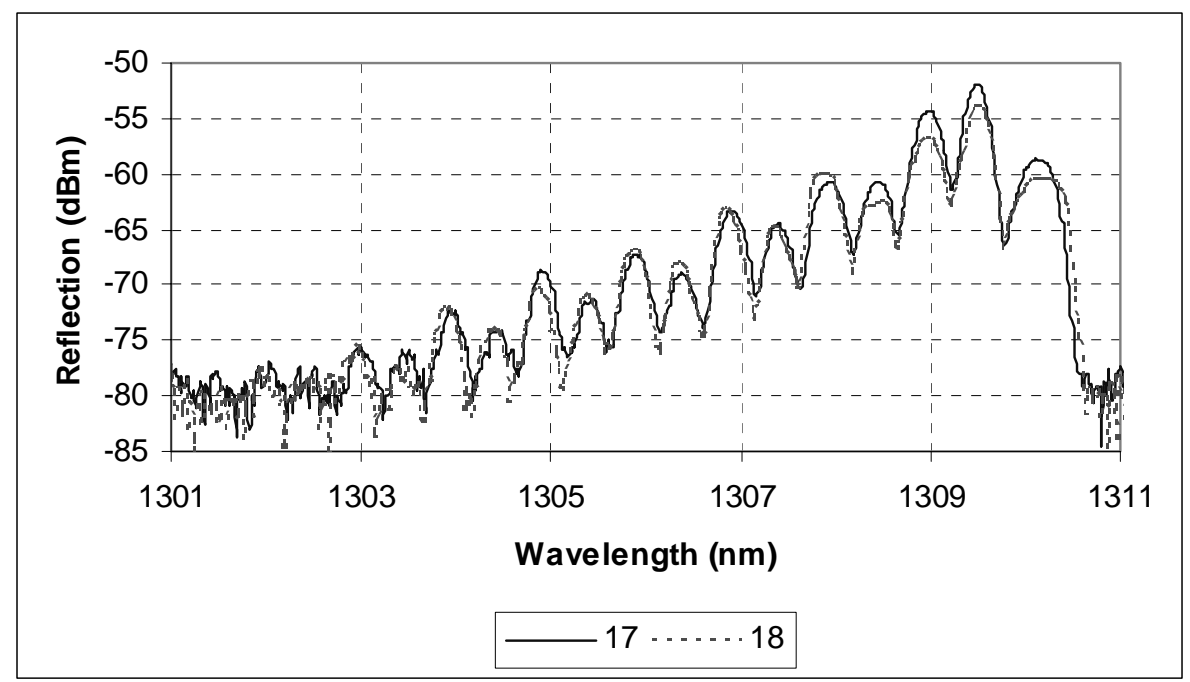

Figure 3. The spectra of the MMFBGs. \#18 acted as a sensing grating and the \#17 as the reference. 


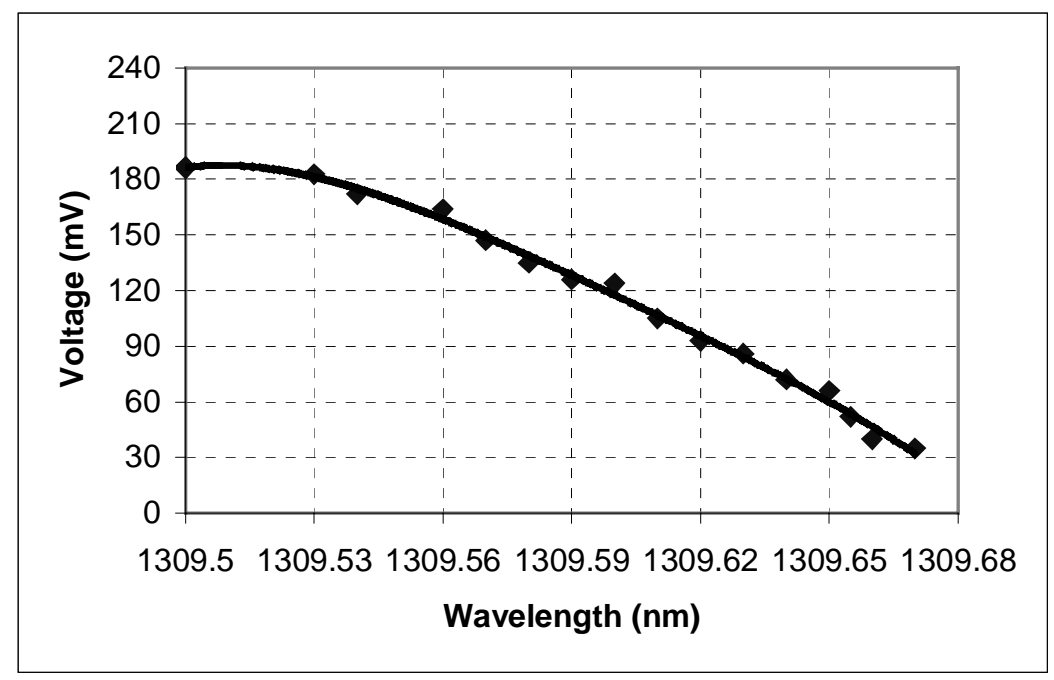

Figure 4 The experimental results showing the relationship between the sensing grating peak wavelength and the detected signal.

The spectra of two MMFBGs with their fundamental mode wavelengths at around 1549nm are shown in Figure 5. The number of distinctive Bragg peaks was smaller than the $1310 \mathrm{~nm}$ gratings, this was because less number of modes were excited due to the use of the single mode fiber pigtailed LED. The MMFBG \#8 was used as a sensing FBG and \#4 as a reference grating.

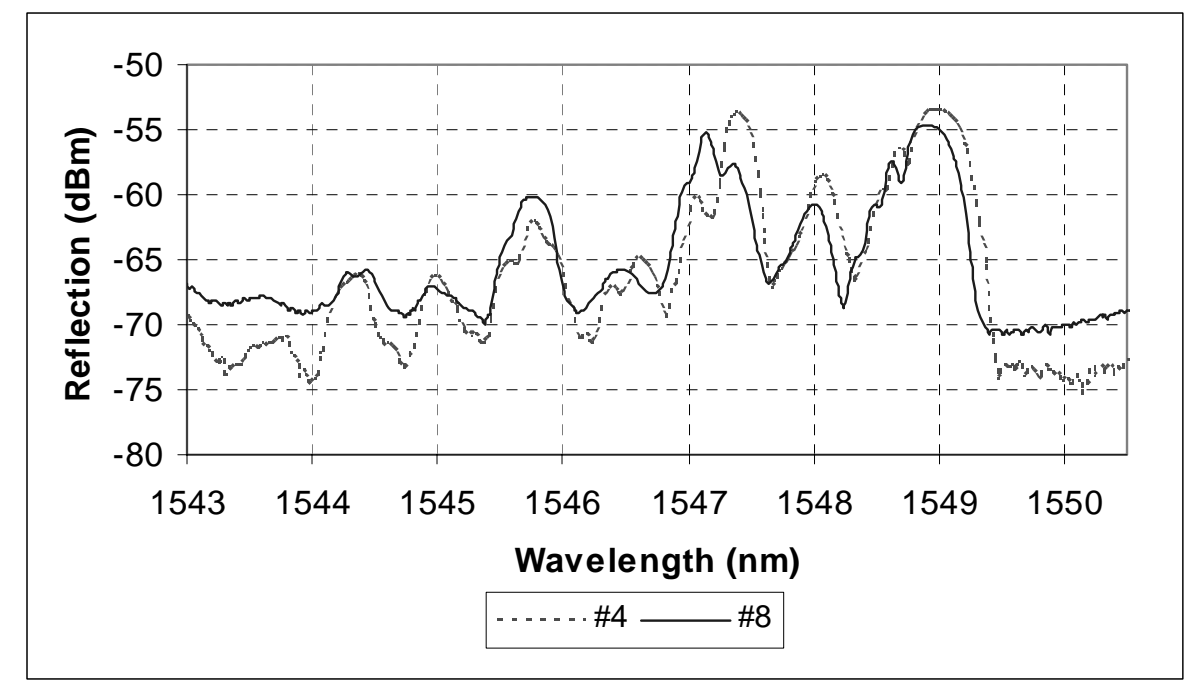

Figure 5 Spectra of two matched MMFBGs at around 1549nm.

When the sensing grating was stretched, its wavelengths increased. As a result, the overlapping area between the sensing and the reference grating decreased. Figure 6 showed a reasonably linear relationship between the sensing wavelength and the detection power. The smaller number of the resonant peaks in the $1550 \mathrm{~nm}$ grating spectra could be responsible for the apparently larger linear range than that of the $1310 \mathrm{~nm}$ gratings. 


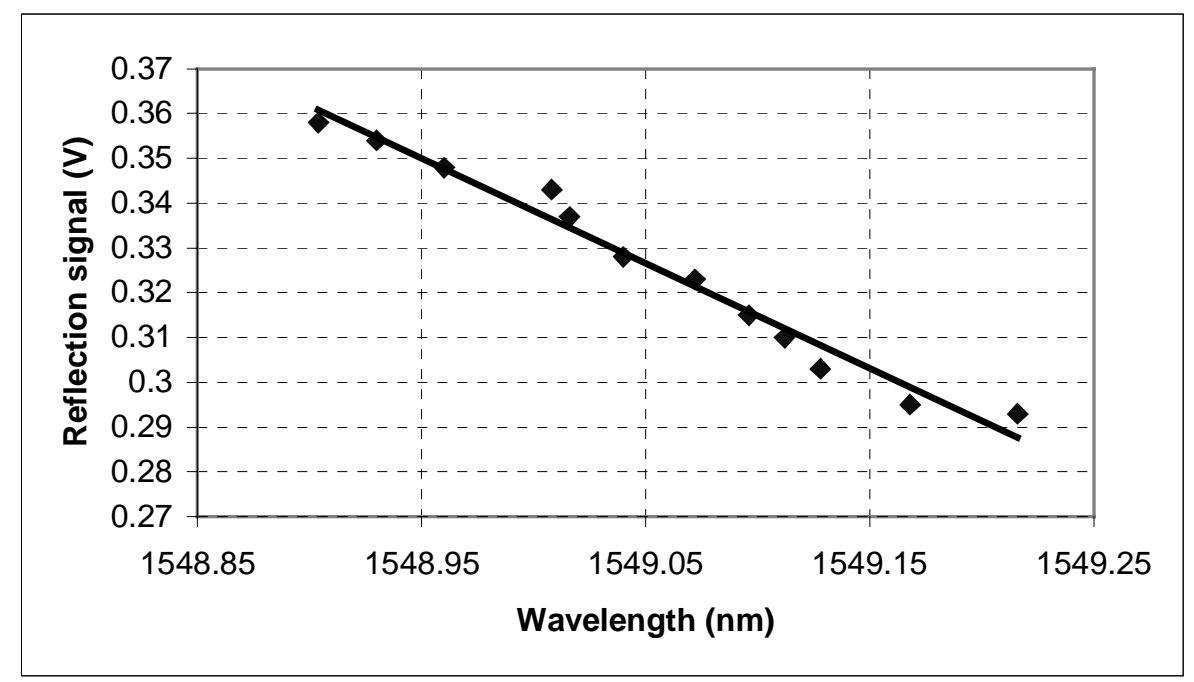

Figure 6 The experimental results from 1549nm MMFBGs showing the relationship between the reflection signal and the sensor FBG wavelength.

A complementary detector could have been implemented to detect the transmission signal of the second coupler which was proportional to the overall sensing grating reflection power. Signal of the additional detector can be used to compensate for the light source, fiber leads instability and improve the system stability. When the MMFBG sensing grating was stretched to shift over the period of the reflection structure, which was approximately $0.5 \mathrm{~nm}$ for the 1310 $\mathrm{nm}$ grating and $0.8 \mathrm{~nm}$ for the $1550 \mathrm{~nm}$ grating, which will result in a corresponding system response. Ongoing work is being carried out to investigate this effect.

\subsection{The fused coupler edge filter scheme}

The spectrum of the over coupled single mode fiber fused coupler is shown in Figure 7. The monotonic slope at around $1550 \mathrm{~nm}$ region suggested that it could be used as an edge filter. The transmission response of the other output arm of the coupler will have an opposite transmission slope. The response of the detection system to strain applied to the sensing grating is plotted in Figure 8. The non-linearity is partially attributed to the response of the filter and partially due to the control of the test jig where the sensing MMFBG could be loose at lower tension level. The linearity was expected to be improved if light was detected at both the output arms of the edge filter coupler and appropriate algorithm was used to correlate with strain.

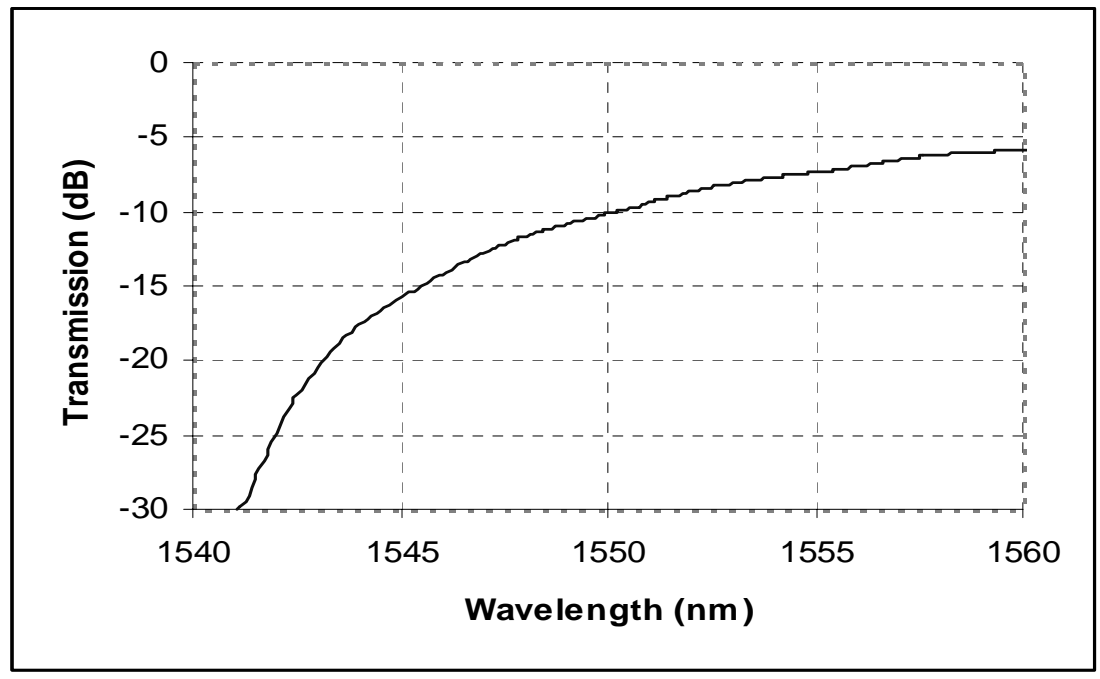

Figure 7 The transmission characteristics in the region around $1550 \mathrm{~nm}$ of an edge filter made of a fused single mode coupler filter. 


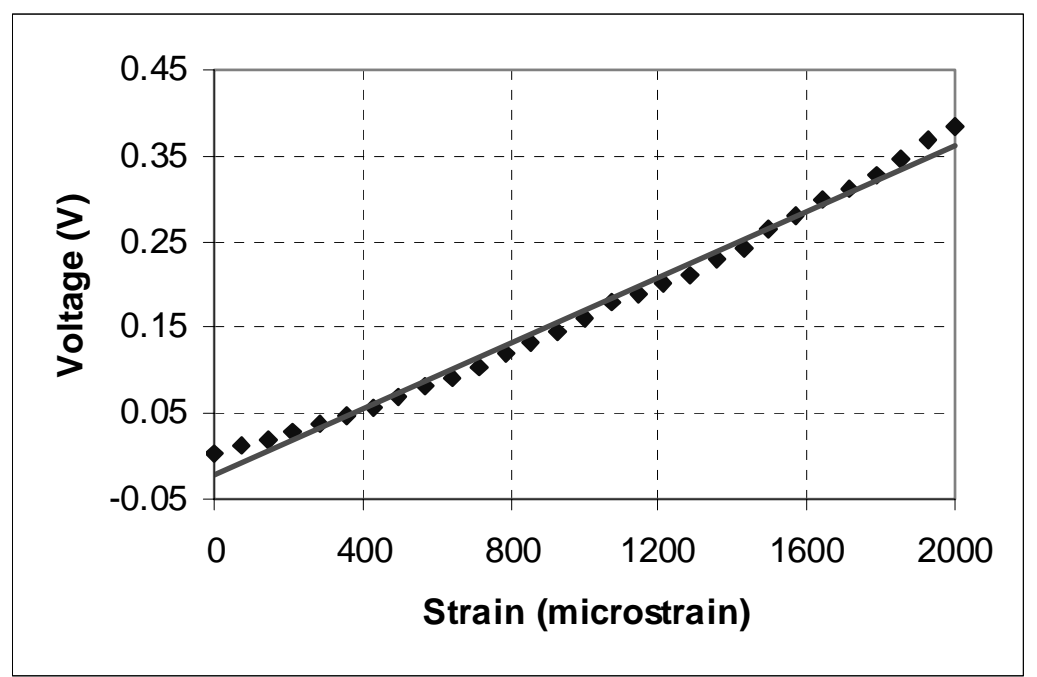

Figure 8 Experimental results showing the output signal as a function of strain applied to the MMFBG.

\section{SUMMARY}

We have described two detection schemes for the MMFBG sensors. The $1^{\text {st }}$ scheme was based on a matched grating the second was based on fused coupler edge filter. For the first scheme we evaluated gratings with fundamental wavelengths at $1310 \mathrm{~nm}$ and $1550 \mathrm{~nm}$ respectively. We have demonstrated that it is feasible to detect wavelength shift of the MMFBG with low cost multimode pigtailed LED light sources. The second scheme was based on a fused, over coupled single mode coupler. The operation range of the later is generally larger than that of the former, but less sensitive. These two approaches may be applied for different applications depending on the requirement of the detection sensitivity and the range of wavelength shift.

\section{REFERENCES}

1. A. F. Fernandez et al. "Fiber Bragg grating temperature sensors for harsh nuclear environments", Proc. 15 $5^{\text {th }}$ Optical Fiber Sensors Conference, 63-66, IEEE, Portland, OR, USA, 2002.

2. A. D. Kersey, T. A. Berkoff, and W. W. Morey, "High-resolution fiber-grating based strain sensor with interferometric wavelength-shift detection", Electronics Letters, 28, 236-238, 1992 .

3. M.D. Todd, B. A. Althouse, G. A. Johnson, and S. T. Vohra, "Performance of elastic beam fiber Bragg grating accelerometers", Proc. OFS-13, 545-548, IEEE, Kyongju Korea, 1999.

4. P. Swart, B. M Laquet, and A. A. Chtcherbakov, "Chirped fiber optic Bragg grating esophageal pressure sensor", Proc. 15 $5^{\text {th }}$ Optical Fiber Sensors Conference, 235-238, IEEE, Portland, OR, USA, 2002.

5. Sheng, H.-J, Ming-Yue Fu, Tzu-Chiang Chen , Wen-Fung Liu and Sheau-Shong Bor, "A lateral pressure sensor using a fiber Bragg grating”, Photonics Technology Letters, IEEE, 16, 1146-1148, 2004.

6. M. G. Xu, L. Reekie, Y. T. Chow and J. P. Dakin, "Optical fiber sensor for high pressure measurement using an infiber grating", Proc. OFS-93, 27-30, 1993.

7. P. M. Cavaleiro, F. M. Araujo, and A. B. Lobo Ribeiro, "Metal-coated fiber Bragg grating sensor for electric current metering," Electronics Letters, 34, 1133-1135, 1998.

8. R. M. Measures, M. M. Ohn, S. Y. Huang, J. Bigue and N. Y. Fan, "Tunable laser demodulation of various fiber Bragg grating sensing modalities", Smart Materials and Structures, 7, 237-247, 1998. 
9. M. A. Davis, A. D. Kersey, J. Sirkis and E. J. Friebele, "Shape and vibration mode sensing using a fiber optic Bragg grating array", Smart Materials and Structures, 5 759-765, 1996.

10. S. M. Melle, K. Liu and R. M. Measures "A passive wavelength demodulation system for guided-wave Bragg grating sensors" IEEE Photonics Technology Letters, 4, 516-18, 1992.

11. Y. Liu, L. Zhang and I Bennion, "Fabricating fiber edge filters with arbitrary spectral response based on tilted chirped grating structures", Measurement Science and Technology 10, L1-L3, 1999.

12. Q. Zhang, D. A. Brown, H. Hung, J. E. Townsend, M. Chen, L. J. Reinhart and T. F. Morse "Use of highly overcoupled couplers to detect shifts in Bragg wavelength" Electronics Letters 31, 480-2, 1995.

13. M. A. Davis and A. D. Kersey, "Matched-filter interrogation techniques for fiber Bragg grating arrays", Electronics Letters, 31, 822-824, 1995.

14. P. Zhang, "High-resolution Photon Counting-OTDR Based Interrogation of Multiplexing Broadband FBG Sensors", PhD Dissertation, Virginia Polytechnic Institute and State University, USA, 2003.

15. K. H. Wanser, K. F. Voss, and A. D. Kersey, "Novel fiber devices and sensors based on multimode fiber Bragg gratings", Proc. SPIE 2360, 265-268, 1994.

16. T. Mizunami, T. Niiho, and T. V. Djambova, "Optical fiber Bragg gratings for fiber-optic bending sensors", Proc. OFS-13, 216-219, IEEE, Kyongju Korea, 1999.

17. W. Zhao and R. O. Claus, "Optical fiber grating sensors in multimode fiber", J. Smart Materials and Structures, $\mathbf{9}$, 212-214, 2000.

18. J. Lim, Q. Yang, B. E. Jones and P. R. Jackson, "Strain and temperature sensors using multimode optical fiber Bragg gratings and correlation signal processing", IEEE Transactions on Instrumentation and Measurement, 51, 622-627,2002. 